\section{A FRACTAL UNIVERSE AND THE IDENTITY OF INDESCERNIBLES}

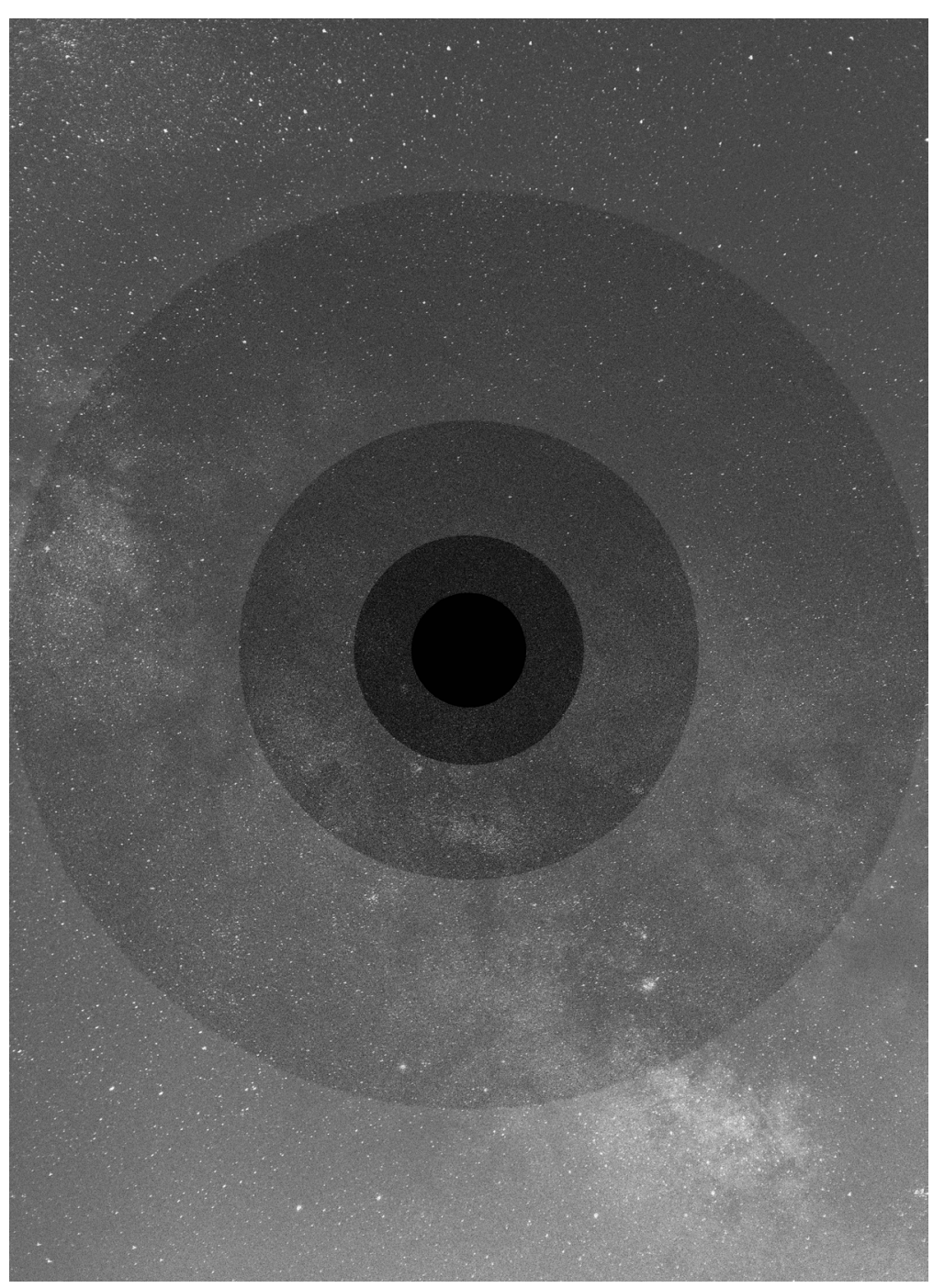

MATTEO CASAROSA

\section{ABSTRACT}

The principle of Identity of Indiscernibles has been challenged with various thought experiments involving symmetric universes. In this paper, I describe a fractal universe and argue that, while it is not a symmetric universe in the classical sense, under the assumption of a relational theory of space it nonetheless contains a set of objects indiscernible by pure properties alone. I then argue that the argument against the principle from this new thought experiment resists better than those from classical symmetric universes three main objections put forth against this kind of arguments.

\section{INTRODUCTION}

The principle of Identity of Indiscernibles is usually formulated as follows: if, for every property $F$, object $x$ has $F$ if and only if object $y$ has $F$, then $x$ is identical to $y .{ }^{1}$ It can be written in the notation of symbolic logic as:

$$
\forall F(F x \leftrightarrow F y) \rightarrow x=y \text {, where } F \text { is a property. }
$$

However, there are in fact several versions of the principle, corresponding to different classes of properties. Not all versions of the principle are controversial. Consider the distinction between pure and impure properties. ${ }^{2}$ Impure properties make reference to particular substances (e.g. being the wife of Socrates) while pure properties do not (e.g. being a wife). If one allows impure properties into the class considered, then the principle seems trivially true: for example, the impure property being distinct from $y$ will certainly discern any $x$ from $y^{3}$.

There is an ongoing debate regarding discernibility through pure properties. Although in recent times the discussion has included empirical arguments, ${ }^{4}$ one of the main objections to the principle being necessarily true is based on thought experiments that present a symmetric universe in which the principle is apparently violated. ${ }^{5}$ A symmetric universe is a possible universe composed of some intrinsically indistinguishable objects, arranged in such a way that

Peter Forrest, "The Identity of Indiscernibles," in Stanford Encyclopedia of Philosophy, last modified August 15, 2010, https://plato.stanford.edu/ entries/identity-indiscernible/.

2 For a detailed discussion of the distinction see e.g. Gary S. Rosenkrantz, "The pure and the impure," Logique et Analyse 22, no. 88 (1979): 515

3 Max Black, "The Identity of Indiscernibles," Mind 61, no. 242 (1952): 15364 considers this same property and concludes that some forms of the principle really are tautological.

4 Steven French, "Identity and Individuality in Quantum Mechanics," Stanford Encyclopedia of Philosophy, last modified August 3, 2015, https://plato. stanford.edu/entries/qt-idind/.

5 The first argument of this kind has been presented in Black, "The Identity of Indiscernibles." The author presented a universe containing only two spheres of the same size. 
all spatial relations among them are mutual-for example, a universe composed of just three qualitatively identical spheres situated at the vertices of an equilateral triangle. Responses to this argument are generally based on some sort of reinterpretation of the visual and spatial construction proposed in the thought experiment.

In this paper, instead of a symmetric universe, I describe a fractal universe and argue that-while there are asymmetric relations among the objects that compose that universe that would seem to make them discernible, and it is not in fact a symmetric universe in the classical sense - under the assumption of a relational theory of space it nonetheless contains a set of objects indiscernible by pure properties alone. I then consider three remarkable objections grounded in thought experiments according to which a symmetric universe is reinterpreted as (i) a non-Euclidean universe comprised of just one object, (ii) one multilocated object, and (iii) comprised of one extended simple object. I aim to show that the first objection does not apply at all to this fractal universe and that the other two are made less plausible due to considerations regarding complexity.

\section{THE THOUGHT EXPERIMENT}

Imagine infinitely many concentric (i.e. having the same center) rings. The radii of these rings increase and decrease without bound. Each one has radius half of that of the one which immediately surrounds it and double of that of the one which it immediately surrounds.

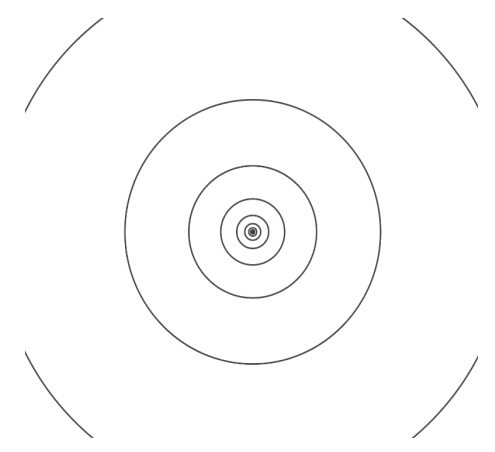

Fig. 1

Now, these rings are, relationally speaking, all in the same situation. Every one of them is inscribed into infinitely many rings, and every one of them contains infinitely many rings. The ratio of one ring to another cannot differentiate them. This is in fact a fractal universe: it appears the same independently of the scale at which you look at it.
It would seem, however, that they are all discernible through pure properties alone, since they are all different in size, and size seems to be analyzable without reference to any particular substance-i.e. it seems to be a pure property. However, under a relational theory of space, there is no absolute size; every measure is relative to other measures. ${ }^{6}$ It would make no sense to think of a universe identical to our own except in size. Under such a theory size is in some sense an extrinsic property, and therefore it is not a pure property if the other entities to which it makes reference are particular substances (i.e. this ring rather than $a$ ring).

I have already laid out some considerations in defense of the thesis that all the rings in this fractal universe are indiscernible through pure properties alone; in the following section I will consider some other plausible objections and build a more formal defense of the thesis.

\section{INDISCERNIBILITY, ASYMMETRY AND AUTOMORPHISMS}

There is certainly some important difference between this fractal universe and symmetric universes presented in previous discussions of the Identity of Indiscernibles. In fact, the fractal universe is not symmetric at all in the obvious sense. Consider any couple of rings: one of them is inside the other, and "being inside" is an antisymmetric relation. ${ }^{7}$ They all would seem therefore to be discernible. Still, that does not prove that they are discernible by means of pure properties alone, for "being inside $X_{k}$," where $X_{k}$ is a particular ring, is not a pure property, since it is plainly analyzed in terms of a particular object-namely, $X_{k}$. Moreover, if we were to "loosen" this property to make it pure, it would no longer differentiate the rings, since "being inside another ring" is common to them all. The fractal universe is not symmetric in the classical sense; however, if we consider the set of all rings in the universe as the domain of a formal language, it appears to be symmetric according to a kind of symmetry described by Caulton and Butterfield. ${ }^{8}$ They call an automorphism (or even, in fact,

- lan Hacking "The Identity of Indiscernibles," The Journal of Philosophy, 72, no. 9 (1975): 249-56.

7 David H. Sanford, "The Problem of the Many, Many Composition

Questions, and Naive Mereology," Noûs 27, no. 2 (1993): 219-28; Shieva Kleinschmidt, "Multilocation and Mereology," Philosophical Perspectives 25, no. 1 (2011). As intuitive as it can be, this may not be completely uncontroversial. Sanford cites a possible counterexample from a novel by Jorge Luis Borge: "I saw the Aleph from all points; I saw the earth in the Aleph and in the earth the Aleph once more and the earth in the Aleph." Aleph and in the earth the Aleph once more and the earth in the Aleph."
Kleinschmidt offers a scenario in which a time-traveling wall ends up being one of its own bricks.

8 Adam Caulton and Jeremy Butterfield, "On Kinds of Indiscernibility in Logic and Metaphysics," British Journal for the Philosophy of Science 63, no. 1 
symmetry) of a domain $D$ a permutation of the domain under which the extensions of all the predicates are invariant. That is, a one-to-one, surjective function $f$ from $D$ to $D$ such that for all $n$, for all predicates $P$ of arity $n$ and for all $b_{1}, \ldots, b_{\mathrm{n}} \in D$ :

$$
P\left(b_{1}, \ldots, b_{n}\right) \leftrightarrow P\left(f\left(b_{1}\right), \ldots, f\left(b_{n}\right)\right)
$$

It seems that the fractal universe has non-trivial automorphisms. For example, the permutation which maps each circle to the one immediately surrounding it will do the job. The reason for this is just the fact that it is a fractal; therefore, it should appear the same regardles of scale, regardless of which ring you chose as your point of reference, so to speak. Each one of these automorphisms intuitively corresponds to a zoom.

This last point, I argue, shows that any two rings are indiscernible through pure properties alone. We can postulate that every pure property of some object $x$ corresponds to a sentence true "of" $x$. In other words, we can assume that for all pure properties $p$ and for all entities $a$ there exists a predicate $P$ and quantifiers $Q_{1}, \ldots, Q_{n}$ such that

$$
\text { a has } p \equiv Q_{1} x_{1}, \ldots, Q_{n} x_{n} P\left(a, x_{1}, \ldots, x_{n}\right)
$$

Now, we are ready for a theorem. Let $a$ be an element of $D$, let $P$ be a predicate, let $Q_{1}, \ldots, Q_{n}$ be quantifiers and let $f$ be an automorphism of $\mathrm{D}$; then we have:

$$
Q_{1} x_{1}, \ldots, Q_{n} x_{n} P\left(a, x_{1}, \ldots, x_{n}\right) \leftrightarrow Q_{1} x_{1}, \ldots, Q_{n} x_{n} P\left(f(a), x_{1}, \ldots, x_{n}\right)^{9}
$$

We have in fact proved that $a$ and $f(a)$ have the same pure properties, or at least the same pure properties corresponding to first order sentences. However, if we broaden the definition of automorphism to higher order predicates, a similar theorem holds for higher order sentences.

\section{CONFRONTING OBJECTIONS TO SYMMETRIC UNIVERSES}

There are three main objections to thought experiments regarding symmetric universes against the Identity of Indiscernibles.

The first is what we might call the Non-Euclidean Space objection. Ian Hacking considers several thought experiments about couples of allegedly distinct but indiscernible objects and insists that they all can be reinterpreted as involving just one object. ${ }^{10}$ Most readers interpret Hacking as suggesting that any symmetric universe made of a couple

(2012): 27-84. This classical sense seems to require every binary relation

to be symmetric.

9 This can be proved by induction on the arity of $P$.

10 lan Hacking, "A Leibnizian Space," Dialogue 14, no. 1 (1975): 89-100, doi: $10.1017 /$ S00122173000456 of allegedly distinct objects could be interpreted as a non-Euclidean universe (i.e. a universe violating some laws of Euclidean geometry, which can be imagined as having bent space) with just one object inside of it. ${ }^{11}$ Max Black's two spheres, for example, would be empirically undistinguishable from just one sphere in cylindric space.

It seems that a cylindric space reinterpretation does not fit well with the fractal universe I have described. In Black's thought experiment, if you want to go from one sphere to the other, there is just one direction you can travel in. In our universe of concentric rings, you can move radially in any direction and you will always meet another ring. If you want to argue that those are all the same ring, you are committed to the claim that the space they inhabit is bent not just in one direction but in any direction. Therefore, a cylindric space would not help, nor would many others; however, one may wonder whether a spherical one could. There are, however, empirical differences between the Euclidean depiction of the fractal universe we have naturally imagined and what would be the case under this tentative reinterpretation in spherical geometry. Quite simply, the rings would not be Euclidean circles. Every circle in Euclidean space has circumference $\Pi$ times its diameter. Circles in spherical space, on the contrary, never instantiate that ratio; rather, the ratio between one spherical circumference and its diameter varies but is always less than $\Pi$ and more than two. We can therefore make explicit that the rings in the fractal universe are Euclidean circles; this would leave that universe conceivable. In fact, I am quite confident that most readers had conceived it as Euclidean from the beginning.

Another reason can be given for the conclusion that the rings cannot be identified one with the other-namely, the discernibility between the exterior and the interior region defined by each ring. Imagine an observer traveling outwards from one ring to another. The observer can ascertain, when he crosses the outer ring, that he is crossing a ring from the inside. (Since the ring is an Euclidean circle, it appears nearer on both sides than it is in front of the observer). The observer can therefore infer that the ring he has reached is not the same he left moving outwards.

What I have offered here is not a rigorous proof that no nonEuclidean space can allow us to reinterpret the rings as just one ring, which would require extensive mathematical work, but I believe it is a good informal argument for that conclusion. The conclusion would completely undermine the Non-Euclidean Space objection; it would not just make it less persuasive as a metaphysical interpretation of the

11 Katherine Hawley, "Identity and Indiscernibility," Mind 118, no. 469 (2009): 101-19. 
qualitative facts described but rather excludes it altogether.

I will now consider the other objections together, because my rebuttals to the two of them are very similar.

O'Leary-Hawthorne argues that bundle theory suggests reinterpreting Black's two spheres as one multilocated sphere. ${ }^{12}$ Let us call this the Multilocation objection.

Hawley suggests redescribing sets of indiscernible objects as one extended simple object, contrary to the intuition that a simple extended object must have a connected location..$^{13}$ Let us call this the Extendedsimple objection.

Both attempts face some difficulties which have been highlighted in the literature; my present aim, however, is just to show that the fractal universe resists both objections better than previous thought experiments. The rationale behind the claim is simplicity.

The argument goes as follows: both multilocation and disconnected extended simples are somehow suspect. If confronted with that which at first seems to be a pair of distinct objects, you would and should require some evidence before you conclude that they are really either the same, multilocated object or a disconnected extended simple. There seems to be a presumption that they are distinct; after all, even the possibility of multilocation and disconnected extended simples is not obvious, while the existence of multiple distinct being seems undeniable, pace Parmenides.

However, if confronted with such a situation you might, despite the presumption, grant non-negligible plausibility to both the multilocation and the extended simple hypotheses.

Now, what if you were confronted with infinitely many seemingly distinct objects? Would not it be infinitely more unbecoming to suppose that there is just one entity? After all the multilocation or extended simple objects, if applied to a couple of objects, would correspond to one disputable identity claim. The same strategies, if applied to what seems to be an infinite multitude, would correspond to an infinite conjunction of such claims.

Moreover, if we want to allow that seemingly distinct rings could really be just one entity, by way of multilocation or extended-simpleness, conflating all of them would be just one possibility among manymany of which would not eliminate the indiscernibility. Suppose, for example, that there were just two multilocated rings, which occupy concentric circular regions alternately. They would be indiscernible by

12 John Hawthorne, "The Bundle Theory of Substance and the Identity of Indiscernibles," Analysis 55, no. 3 (1995): 191-96.

13 Hawley, "Identity and Indiscernibility," 101-19; Ned Markosian, "Simples,"

Australasian Journal of Philosophy 76, no. 2 (1998): 213-28. pure properties alone, for reasons similar to those mentioned for the initial interpretation with infinitely many rings. Therefore, one needs something more specific than a claim like "In the imagined universe there is a multilocated object," or "In the imagined universe there is a disconnected extended simple object."

These considerations are meant to highlight that both objectionsif applied to the fractal universe-result in very complex and specific claims, which would be unbecoming unless one has strong, independent reasons to favor that interpretation over the natural one, which involves infinitely many indiscernible rings.

This response does not show that the multilocation objection and the extended simple have no force against the new thought experiment, as was the case for the Non-Euclidean Space objection, but it nevertheless shows that they face further difficulties other than those already present in previous symmetric universes.

\section{CONCLUSION}

The Principle of Identity of Indiscernibles has been attacked in the past with several thought experiments, which have usually been considered inconclusive. The same set of empirical facts described in the thought experiments can usually be given many different metaphysical interpretations, and it is not easy to say which should be preferred. In this paper, I have presented a new thought experiment whose geometric features secure it against a kind of reinterpretation, and I have defended it against other objections using arguments from theoretical simplicity.

Finally, I must point out that my argument depends on a few controversial assumptions. One is the relational theory of space which, however, seems to be implicitly assumed in many discussions of the Principle; the other, less controversial assumption is the possibility of an infinite multitude of objects. The second commitment is perhaps the most curious. Previous arguments for and against the Principle have no connection with that proposition. If one finds all my other premises true, and still believes the Principle of Identity of Indiscernibles should not be abandoned, they could use the Principle to argue for finitism. Future research could make this an argument worthy of consideration. 


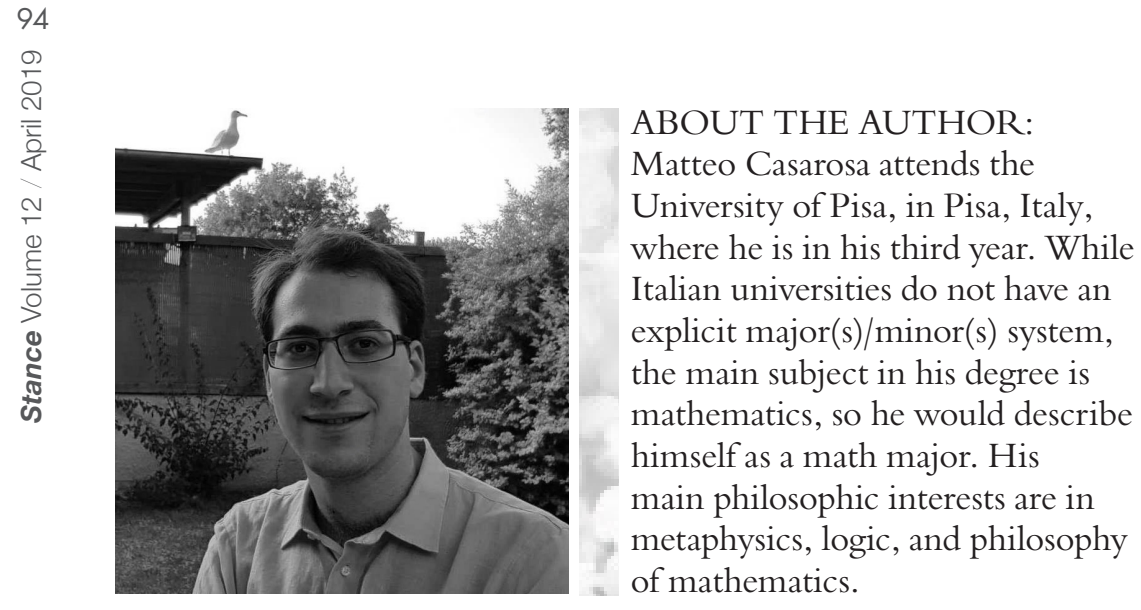

of mathematics. 